\title{
Exposure to Childhood Maltreatment as Related to Adult Intimate Partner Victimization among College Students: The Contribution of Childhood Polyvictimization
}

\author{
LaVerne McQuiller Williams, Judy Porter, 0. Nicholas Robertson \\ Rochester Institute of Technology, Rochester, USA \\ Email: jlpgcj@rit.edu
}

How to cite this paper: McQuiller Williams, L., Porter, J., \& Robertson, O. N. (2021). Exposure to Childhood Maltreatment as Related to Adult Intimate Partner Victimization among College Students: The Contribution of Childhood Polyvictimization. Psychology, 12, 25-42.

https://doi.org/10.4236/psych.2021.121002

Received: December 9, 2020

Accepted: January 8, 2021

Published: January 11, 2021

Copyright ( 2021 by author(s) and Scientific Research Publishing Inc. This work is licensed under the Creative Commons Attribution International License (CC BY 4.0).

http://creativecommons.org/licenses/by/4.0/

(c) (i) Open Access

\begin{abstract}
This study will explore polyvictimization as a child and the effects of this on adult intimate personal violence. The study consisted of 260 surveys of participants at a northeastern middle size university. Cross tabulations and binomial regressions were conducted. The current study had two objectives: 1) to examine the extent of partner victimization and child maltreatment (witnessing intra-parental abuse and/or experiencing child abuse/sexual abuse) among a sample of college students and determine whether these experiences vary by gender; 2 ) to explore whether polyvictimization, including child maltreatment, may increase the risk of intimate partner victimization in adulthood. Findings revealed that women were statistically significantly more likely than men to report experiencing higher rates of psychological, physical and sexual abuse as an adult, although findings indicated men were also victimized by a partner within the last year. A large number of respondents reported experiencing maltreatment as a child, which included physical abuse, sexual abuse, or witnessing abuse by a parent/caregiver, although findings in the current study revealed no significant differences between men and women. The results also found some support for the effects of polyvictimization and adult intimate partner victimization. Implications and directions for further research are discussed. It is suggested that institutions develop means to educate and address these concerns for all genders. Parents/caregivers and health professionals should be made aware of effects on adults' experiences of intimate partner violence that violence witnessed as a child and abuse experienced as a child may have.
\end{abstract}

\section{Keywords}

Intimate Partner Violence, Child Maltreatment, Polyvictimization, Gender, 


\section{Introduction}

Experiencing violence by an intimate partner is a reality for a substantial number of college students. Several studies found that nearly one-third of college students experience violence in a dating relationship (Gover, Kaukinen, \& Fox, 2008; Orcutt, Garcia, \& Pickett, 2005; Sabina \& Straus, 2008). Since intimate partner violence (IPV) among college students occurs at alarmingly high rates, it is important to understand the factors that may increase the risk of victimization. One possible risk factor is child maltreatment. Child maltreatment is one of the most commonly studied risk factors for intimate partner violence (Jennings et al., 2011, Riggs, Caufield, \& Field, 2009). Both witnessing inter-parental violence and experiencing child abuse have been found to increase the likelihood of victimization among college students (Langhinrichsen-Rohling et al., 2004). One focus of the present study was to examine whether witnessing intraparental abuse and experiencing childhood abuse was associated with adult intimate partner victimization.

Children and teens experience high rates of physical, sexual, and emotional maltreatment by caregivers (Aakvaag, Thoresen, Wentzel-Larsen, \& Dyb, 2017; Finkelhor, Turner, Shattuck, \& Hamby, 2015; Peterson, Florence, \& Klevens, 2018). In 2016, 1750 children died nationwide due to maltreatment and another 676,000 suffered child maltreatment (U.S. Department of Health \& Human Services, 2018). Survey data from the National Survey of Children's Exposure to Violence indicate that $15 \%$ of children and teens experienced at least one form of child maltreatment in the previous year (Finkelhor et al., 2015). Child maltreatment has been linked with a wide range of adverse outcomes in adulthood including substance abuse, depression, cognitive difficulties, and interpersonal problems (Colman \& Widom, 2004; Herrenkohl et al., 2015; Stevens et al., 2018; Widom et al., 2012).

There has been increasing interest in the link between child maltreatment and adult victimization, especially by an intimate partner (Bensley, Van Eenwyck, \& Wynkopp Simmons, 2003; Becker, Stuewing, \& McCloskey, 2010; Daigneault, Hebert, \& McDuff, 2009; Millett, Kohl, Jonson-Reid, Drake, \& Pretra, 2013; Renner \& Slack, 2006). Studies have found that individuals exposed to child maltreatment are more likely to be victimized in adulthood by an intimate partner than their non-maltreated peers (Gover et al., 2008; Langhinrichsen-Rohling, Hankla, \& Stormberg, 2004; Widom, Czaja, \& Dutton, 2014). Bensley, Van Eenwyck, and Wynkopp Simmons (2003) found that women who experienced childhood abuse or witnessed parental violence in childhood were six times more likely to experience physical partner violence as adults. A more recent study, using a U.S. national sample, found that for women, physical and psychological abuse in childhood was significantly related to adult partner victimi- 
zation for women (Richards, Tillyer, \& Wright, 2017).

Posited within social learning theories (Akers, 1998; Bandura, 1973, 1977), these studies suggest a causal relationship between prior victimization via the "cycle of violence", whereby "children raised in a violent home, through processes of observational learning, modeling, and direct behavioral conditioning, will come to see violence as an appropriate response to conflict" (Smith-Marek et al., 2015: p. 499). According to this perspective, individuals who experience abuse in the family of origin may be more likely to accept violence as an expected and normative facet of interpersonal relationships (Gover, Kaukinen, \& Fox, 2008). Many studies have found empirical associations between family of origin violence and an increased risk of later intimate partner victimization (Brownridge, 2006; Foshee et al., 2005; Gomez, 2011; Hines, 2007; Kaukinen, Buchanana, \& Gover, 2015; Stith et al., 2000).

Despite the large body of work on child maltreatment and subsequent abuse in adulthood, one limitation is that the majority of literature examining child maltreatment focuses on one category of maltreatment (Richards, Tillyer, \& Wright, 2017; Richmond et al., 2009). This is problematic as research has shown that child maltreatment victimization experiences accumulate leading to a higher risk of victimization into adulthood (Desai, Arias, \& Thompson, 2002; Finkelhor, Ormond, \& Turner 2007a, 2007b; Ports, Ford, \& Merrick, 2016).

Polyvictimization, defined as the exposure to high levels and multiple forms of violence (e.g., witnessing parental abuse, sexual abuse, and neglect), may have even more harmful effects on victims than single forms of maltreatment (Finkelhor et al., 2007a). A relatively new concept in the area of interpersonal victimization, polyvictimization was introduced by Finkelhor and colleagues in a 2007 empirical study (Finkelhor et al., 2007a) and has been tested in several exploratory studies (Finkelhor et al., 2007a, 2007b; Ford, Elahi, Connor, \& Freuh, 2010; Ford, Grasso, Hawke \& Chapman, 2013; Sabina \& Straus, 2008). It has been suggested that studies that examine only one category of victimization likely underestimate the true "burden of victimization that young people experience" and "fail[s] to show the interrelationships among different kinds of victimization" (Finkelhor, Ormond, Turner, \& Hamby, 2015: pp. 5-6).

Moreover, while a large body of literature has documented rates of intimate partner victimization among college students in general, previous research has focused largely on the victimization experiences of women college students (e.g., DeKeseredy et al., 2019; Richmond et al., 2009), often ignoring the experiences of men. The few studies that examined victimization among men found that that men and women experience similar rates of psychological (Bookwala et al., 1992; Clark, Beckett, Wells, \& Dungee-Anderson, 1994; Stets \& Henderson, 1991) and physical abuse (Harned, 2002; Marshall \& Rose, 1988) in dating relationships.

\section{Current Study}

The current study contributes to research on interpersonal violence in two ways. First, we examine the extent of partner victimization and child maltreatment 
among a sample of a college students and whether these experiences vary by gender. The majority of studies that examine victimization are often limited to women. Second, this study makes a contribution by exploring whether polyvictimization may increase the risk of subsequent intimate partner victimization in adulthood. This is an important contribution because of the dearth of research on risk factors for intimate partner victimization among college students beyond one form of child maltreatment.

\section{Materials and Method}

\subsection{Data Collection}

Surveys were administered randomly to forty classes at a medium size university in the northeastern United States. This university was selected because the university population was widely dispersed across a unique array of academic programs ranging from liberal arts, STEM programs, and the Fine Arts. The University Institutional Review Board approved the survey prior to its dissemination. A total of 260 survey responses were collected. Respondents were informed that the survey was voluntary with no reward or punishment attached. Students could desist taking the survey at any time and were instructed not to take the survey if they had completed it in a previous class.

\section{Participants}

Among the respondents, 54.6\% (142) indicated that they were female, $45 \%$ (117) male, and one transgender $(0.4 \%)$. The majority of respondents indicated their race or ethnicity as white $(145,55.8 \%)$, with 41 Black not Hispanic (15.8\%), 34 Hispanic or Latino (13.1\%), 25 Asian or Pacific Islander (9.6\%), seven American Indian or Alaskan Native (2.7\%), and seven other (2.7\%). Among males, 72 respondents identified as white (61.5\%), 16 Black not Hispanic (13.7\%), 11 Hispanic or Latino (9.4\%), nine Asian or Pacific Islander (7.7\%), two American Indian or Alaskan Native (1.7\%), six other (5.1\%) and 1 missing (0.9\%). Among females, 73 (51.4\%) identified as white, 24 (16.9\%) as Black not Hispanic, 23 (16.3\%) as Hispanic or Latino, 16 (11.3\%) Asian or Pacific Islander, five (3.5\%) as American Indian or Alaskan Native, and one (0.7\%) as other. The lone respondent $(0.7 \%)$ who identified as transgender indicated Black not Hispanic as their race or ethnicity (Table 1 ).

\subsection{Measures}

The dependent variables are dummy variables created from questions pertaining to victimization as a child and as an adult. Gender is coded 0 for men, 1 for women. To measure child maltreatment various questions asked if some event occurred prior to the age of 18 years old -0 for no and 1 for yes. Psychological abuse included survey questions concerning child maltreatment at the hands of parents or caregivers and witnessing a parent or caregiver abuse the other parent or caregiver. Physical abuse was measured by questions assessing if, as a child, the participant had suffered several forms of physical abuse by their parents or 
Table 1. Cross tabulation gender by race or ethnicity $\mathrm{N}=260$.

\begin{tabular}{|c|c|c|c|c|c|c|c|c|}
\hline \multirow{3}{*}{ Gender } & \multicolumn{7}{|c|}{ Race or Ethnicity } & \multirow[b]{2}{*}{ Total } \\
\hline & $\begin{array}{l}\text { White-not } \\
\text { Hispanic }\end{array}$ & $\begin{array}{c}\text { Black-not } \\
\text { Hispanic }\end{array}$ & $\begin{array}{l}\text { Hispanic } \\
\text { or Latino }\end{array}$ & $\begin{array}{l}\text { Asian or } \\
\text { Pacific } \\
\text { Islander }\end{array}$ & $\begin{array}{l}\text { American } \\
\text { Indian or } \\
\text { Alaskan } \\
\text { Native }\end{array}$ & Other & Missing & \\
\hline & $\mathrm{n} \%$ & $\mathrm{n} \%$ & n \% & $\mathrm{n} \%$ & n \% & n \% & $\mathrm{n} \%$ & $\mathrm{n} \%$ \\
\hline \multirow{2}{*}{ Men } & 72 & 16 & 11 & 9 & 2 & 6 & 1 & 117 \\
\hline & $61.5 \%$ & $13.7 \%$ & $9.4 \%$ & $7.7 \%$ & $1.7 \%$ & $5.1 \%$ & $0.9 \%$ & $100.0 \%$ \\
\hline \multirow{2}{*}{ Women } & 73 & 24 & 23 & 16 & 5 & 1 & 0 & 142 \\
\hline & $51.4 \%$ & $16.9 \%$ & $16.2 \%$ & $11.3 \%$ & $3.5 \%$ & $0.7 \%$ & $0.0 \%$ & $100.0 \%$ \\
\hline \multirow{2}{*}{ Transgender } & 0 & 1 & 0 & 0 & 0 & 0 & 0 & 1 \\
\hline & $0.0 \%$ & $100.0 \%$ & $0.0 \%$ & $0.0 \%$ & $0.0 \%$ & $0.0 \%$ & $0.0 \%$ & $100.0 \%$ \\
\hline \multirow{2}{*}{ Total } & 145 & 41 & 34 & 25 & 7 & 7 & 1 & 260 \\
\hline & $55.8 \%$ & $15.8 \%$ & $13.1 \%$ & $9.6 \%$ & $2.7 \%$ & $2.7 \%$ & $0.4 \%$ & $100.0 \%$ \\
\hline
\end{tabular}

caregivers. Sexual abuse as a child was determined by responses to a question asking if the participant had been forced to engage in vaginal, anal, or oral intercourse with a parent or caregiver.

Psychological and physical abuse as an adult by a partner over the past year was measured with Straus, Hamby, Boney-McCoy, and Sugarman's (1996) Revised Conflict Tactics Scale (CTS-2). To assess psychological abuse, survey questions included questions about the respondent's partner and if the partner had insulted or sworn at them, put them down in front of others, or threatened to hit or throw something at the respondent (Cronbach's Alpha 0.705). Physical abuse was assessed with survey questions concerning having been pushed, grabbed, shoved; having been slapped; having been kicked or bit; having been beat up; having been hit with something; having been choked, and if they had been threatened with a weapon (Cronbach's Alpha 0.802). Sexual abuse was assessed with two questions asking if the respondent had unwanted sexual penetration attempted against them, or if sexual penetration against their will had occurred-these were run independently as the Cronbach's Alpha (0.521) was too low for a scale possibly due to the small number of items.

Intra-parental abuse was measured with questions that asked respondents if as a child they witnessed one parent/caretaker grab, put down, insult, beat up, choke, or threaten the other parent or caretaker (Cronbach's Alpha 0.701). Child maltreatment was assessed with three questions that asked respondents if they had a parent or caregiver who choked, beat up, or sexually abused them-these were run independently as the Cronbach's Alpha was too low for a scale (0.383). A 4-point scale was used for responses-never, once or twice, three to ten times, more than 10 times. Due to sparse responses these items were dichotomized for the sake of analysis with a 0 for no, and 1 for yes. 


\subsection{Data Analysis}

All data analyses including descriptive statistics, cross tabulations, and binomial regression were conducted using IBM SPSS Statistics 21 . All measures were dichotomized with yes or no. Gender was coded 0 for men and 1 for women. Race or ethnicity was coded 0 for white and 1 for being a member of a racial or ethnic minority (REM) group. Binomial regression analyses were conducted with all variables for child maltreatment, child witness of intra-parental abuse, and adult sexual abuse. Scales were used to assess adult psychological and physical victimization. Binomial regression analyses were conducted with variables in a hierarchical position and with the Wald backward stepwise selection method.

\section{Results}

\subsection{Descriptive and Binomial Regression Results}

\section{Childhood Maltreatment and Gender-All Respondents}

Table 2 illustrates via cross-tabulation the number of incidences suffered by children at the hands of their parents or caregivers as well as the number of times as a child they witnessed intra-parental or intra-caregiver abuse. Only gender achieved a statistically significant association with having been a child victim of sexual abuse at the hands of a parent or caregiver. Thirty-eight (15.1\%) of the respondents replied yes to having experienced some form of sexual abuse as a child victim of their parents or caregiver. Of those, the majority were women $(\mathrm{n}=29,20.9 \%)$ while nine $(8.0 \%)$ were men. The table indicated that even without a statistically significant difference between men and women for the bulk of the variables examined, both men and women experienced victimization in the past year. The most prevalent form of childhood maltreatment was psychological abuse in the form of witnessing one parent or caregiver grab, push, or shove the other ( $n=93,37.1 \%)$; followed by insulting or swearing at the other parent/caregiver $(n=91,36.3 \%)$, put the other parent/caregiver down in front of others $(n=86,34.3 \%)$, beat up the other parent/caregiver $(n=39,19.4 \%)$, and choked the other parent/caregiver $(\mathrm{n}=16,6.5 \%)$. Child maltreatment in the form of physical abuse was the second most prevalent form of abuse in the following order: Parent/caregiver beat you up $(n=49,19.5 \%)$, sexually abused you $(\mathrm{n}=38,15.1 \%)$, choked you $(\mathrm{n}=24,9.8 \%)$, or threatened you with a weapon ( $\mathrm{n}$ $=8,3.2 \%)$.

\subsection{Women Respondents}

In order of prevalence, women respondents reported psychological abuse in the form of witnessing intra-parental/caregiver abuse-grabbed, pushed, or shoved $(\mathrm{n}=58,62.4 \%)$ put down $(\mathrm{n}=54,38.8 \%)$, insulted or swore $(\mathrm{n}=53,38.1 \%)$, beat up $(\mathrm{n}=27,19.4 \%)$, or choked $(\mathrm{n}=12,8.8 \%)$. Physical abuse was next in prevalence in reporting. Nearly a quarter of women respondents reported being beaten up by a parent/caregiver $(n=33,23.7 \%)$. Over one-fifth of women respondents indicated they had been forced to have sex (vaginal, oral, or anal 
Table 2. Cross-tabulation child maltreatment by gender $\mathrm{N}=260$.

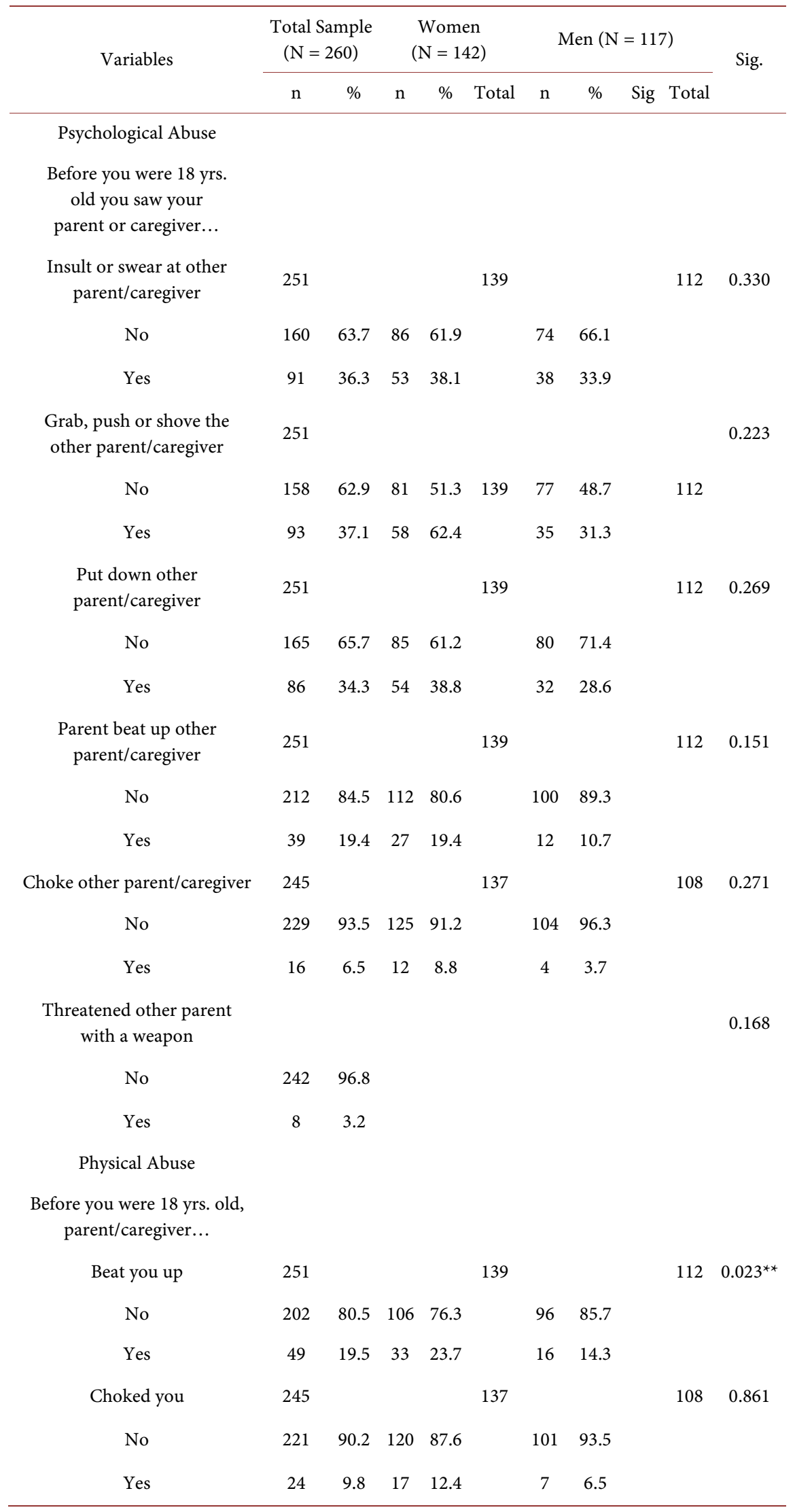




\section{Continued}

\begin{tabular}{ccccccccccc}
\hline Sexually abused you & 251 & \multicolumn{1}{c}{139} & & & 112 & $0.001^{*}$ \\
No & 213 & 84.9 & 110 & 79.1 & 103 & 92.0 & & \\
Yes & 38 & 15.1 & 29 & 20.9 & 9 & 8.0 & & \\
$\begin{array}{c}\text { Threatened you with } \\
\text { a weapon }\end{array}$ & 249 & & & & 137 & & & 112 & $0.040^{* *}$ \\
No & 241 & 96.8 & 130 & 94.9 & 111 & 99.1 & & \\
Yes & 8 & 3.2 & 7 & 5.1 & 1 & 0.9 & &
\end{tabular}

${ }^{*} p \leq 0.001,{ }^{* *} p \leq 0.05$.

intercourse) with a parent/caregiver $(n=29,20.9 \%)$. Chi-Square indicated that women were more likely to report being grabbed, pushed, beaten up, and sexually abused by a parent/caretaker than were men (Table 3 ).

\section{Men Respondents}

The majority of men who responded indicated they had experienced psychological abuse with 38 (33.9\%) reporting seeing their parent or caregiver insult or swear at the other parent/caregiver, 35 (31.3\%), saw the one parent/caregiver grab, push, or shove the other; and $32(28.6 \%)$ witnessed one parent/caregiver put down the other one. Twelve men (10.7\%) saw one parent/caregiver beat up the other one, while four men witnessed one parent/caregiver choke the other parent/caregiver (Table 3).

Sixteen men (14.3\%) reported being beat up as a child by their parent/caregiver. Nine men (8.0\%) reported being sexually abused as a child by their parent/caregiver via oral or anal sexual intercourse. Seven men indicated they had been choked by their parent/caregiver (6.5\%) and one man said he had been threatened with a weapon by his parent or caregiver (0.9\%) (Table 3 ).

\subsection{Adult Intimate Partner Victimization}

A crosstab analysis of adult psychological, physical, and sexual abuse by an intimate partner found that women were more likely to report all forms of abuse than were men. Psychological $(p \leq 0.001)$ abuse, physical $(p \leq 0.022)$ abuse, and attempted unwanted sexual contact $(p \leq 0.008)$ were statistically significant and associated with gender-women were more likely to report psychological and physical abuse, as well as attempted unwanted sexual contact as adults. Eighty-three women $(59.7 \%)$ and 47 men $(43.1 \%)$ reported psychological abuse. Seventy-one women (52.2\%) and 33 men (30.3\%) reported physical abuse. Twenty-four women $(17.3 \%)$ and 10 men $(9.0 \%)$ reported attempted unwanted sexual contact. Twelve women (8.7\%) and four (3.6\%) men reported unwanted sexual contact (Table 4).

Binomial Regression Analyses

Psychological Abuse

A binomial regression analysis found a statistically significant association such that respondents were over nine times as likely (Exp. $\mathrm{B}=9.172, p \leq 0.000)$ to 
Table 3. Cross-tabulation child maltreatment by gender $\mathrm{N}=260$.

\begin{tabular}{|c|c|c|c|c|c|c|c|c|c|c|}
\hline \multirow[t]{2}{*}{ Variables } & \multicolumn{2}{|c|}{$\begin{array}{l}\text { Total Sample } \\
(\mathrm{N}=260)\end{array}$} & \multirow[t]{2}{*}{ Sig } & \multicolumn{2}{|c|}{$\begin{array}{l}\text { Women } \\
(\mathrm{N}=142)\end{array}$} & \multirow[t]{2}{*}{ Total } & \multicolumn{2}{|c|}{$\begin{array}{c}\text { Men } \\
(\mathrm{N}=117)\end{array}$} & \multirow[t]{2}{*}{ Total } & \multirow[t]{2}{*}{ Sig. } \\
\hline & $\mathrm{n}$ & $\%$ & & $\mathrm{n}$ & $\%$ & & $\mathrm{n}$ & $\%$ & & \\
\hline \multicolumn{11}{|l|}{ Psychological Abuse } \\
\hline \multicolumn{11}{|l|}{$\begin{array}{c}\text { Before you were } 18 \text { yrs. } \\
\text { old you saw your } \\
\text { parent or caregiver... }\end{array}$} \\
\hline $\begin{array}{c}\text { Insult or swear at other } \\
\text { parent/caregiver }\end{array}$ & 251 & & 0.330 & & & 139 & & & 112 & 0.491 \\
\hline No & 160 & 63.7 & & 86 & 61.9 & & 74 & 66.1 & & \\
\hline Yes & 91 & 36.3 & & 53 & 38.1 & & 38 & 33.9 & & \\
\hline $\begin{array}{l}\text { Grab, push or shove the } \\
\text { other parent/caregiver }\end{array}$ & 251 & & $0.100^{\star *}$ & & & & & & & \\
\hline No & 158 & 62.9 & & 81 & 51.3 & 139 & 77 & 48.7 & 112 & 0.088 \\
\hline Yes & 93 & 37.1 & & 58 & 62.4 & & 35 & 31.3 & & \\
\hline $\begin{array}{l}\text { Put down other } \\
\text { parent/caregiver }\end{array}$ & 251 & & $0.091^{\star *}$ & & & 139 & & & 112 & 0.088 \\
\hline No & 165 & 65.7 & & 85 & 61.2 & & 80 & 71.4 & & \\
\hline Yes & 86 & 34.3 & & 54 & 38.8 & & 32 & 28.6 & & \\
\hline $\begin{array}{l}\text { Parent beat up other } \\
\text { parent/caregiver }\end{array}$ & 251 & & 0.151 & & & 139 & & & 112 & 0.058 \\
\hline No & 212 & 84.5 & & 112 & 80.6 & & 100 & 89.3 & & \\
\hline Yes & 39 & 19.4 & & 27 & 19.4 & & 12 & 10.7 & & \\
\hline $\begin{array}{l}\text { Choke other } \\
\text { parent/caregiver }\end{array}$ & 245 & & 0.271 & & & 137 & & & 108 & 0.112 \\
\hline No & 229 & 93.5 & & 125 & 91.2 & & 104 & 96.3 & & \\
\hline Yes & 16 & 6.5 & & 12 & 8.8 & & 4 & 3.7 & & \\
\hline $\begin{array}{l}\text { Threaten other } \\
\text { parent/caregiver } \\
\text { with a weapon }\end{array}$ & & & 0.168 & & & & & & & \\
\hline Physical Abuse & & & & & & & & & & \\
\hline \multicolumn{11}{|l|}{$\begin{array}{l}\text { Before you were } 18 \text { yrs. } \\
\text { old, parent/caregiver... }\end{array}$} \\
\hline Beat you up & 251 & & $0.023^{*}$ & & & 139 & & & 112 & 0.060 \\
\hline No & 202 & 80.5 & & 106 & 76.3 & & 96 & 85.7 & & \\
\hline Yes & 49 & 19.5 & & 33 & 23.7 & & 16 & 14.3 & & \\
\hline Choked you & 245 & & 0.284 & & & 137 & & & 108 & 0.121 \\
\hline No & 221 & 90.2 & & 120 & 87.6 & & 101 & 93.5 & & \\
\hline Yes & 24 & 9.8 & & 17 & 12.4 & & 7 & 6.5 & & \\
\hline Sexually abused you & 251 & & $0.001^{\star}$ & & & 139 & & & 112 & $0.005^{*}$ \\
\hline No & 213 & 84.9 & & 110 & 79.1 & & 103 & 92.0 & & \\
\hline Yes & 38 & 15.1 & & 29 & 20.9 & & 9 & 8.0 & & \\
\hline
\end{tabular}




\section{Continued}

\begin{tabular}{cccccccccc}
\hline Threatened to hit you & 249 & & 0.694 & & 137 & & 112 & 0.281 \\
No & 241 & 96.8 & & 130 & 94.9 & 111 & 99.1 & & \\
Yes & 8 & 3.2 & & 7 & 5.1 & 1 & 0.9 & & \\
\hline
\end{tabular}

${ }^{*} p \leq 0.005,{ }^{* *} p \leq 0.10$.

Table 4. Cross-tabulation of adult psychological, physical, and sexual abuse by gender $\mathrm{N}$ $=260$.

\begin{tabular}{|c|c|c|c|c|c|c|c|c|c|}
\hline & \multicolumn{3}{|c|}{$\begin{array}{l}\text { Total Sample } \\
\qquad(\mathrm{N}=260)\end{array}$} & \multicolumn{3}{|c|}{$\begin{array}{l}\text { Women } \\
(\mathrm{N}=142)\end{array}$} & \multicolumn{3}{|c|}{$\begin{array}{c}\text { Men } \\
(\mathrm{N}=117)\end{array}$} \\
\hline & $\mathrm{n}$ & $\%$ & Sig & $\mathrm{n}$ & $\%$ & Sig & $\mathrm{n}$ & $\%$ & Sig \\
\hline Physical Abuse & & & & & & $0.022^{* *}$ & & & \\
\hline No & 118 & 47.6 & & 56 & 40.3 & & 62 & 56.9 & \\
\hline Yes & 130 & 52.9 & & 83 & $59.7^{\star}$ & & 47 & 43.1 & \\
\hline Psychological Abuse & & & & & & $0.001^{*}$ & & & \\
\hline No & 141 & 57.6 & & 65 & 47.8 & & 76 & 69.7 & \\
\hline Yes & 104 & 42.4 & & 71 & $52.2^{*}$ & & 33 & 30.3 & \\
\hline $\begin{array}{l}\text { Attempted Unwanted } \\
\text { Sexual Contact }\end{array}$ & 250 & & & & & $0.008^{\star *}$ & & & \\
\hline No & 216 & 86.4 & & 115 & 82.7 & & 101 & 91.0 & \\
\hline Yes & 34 & 13.6 & & 24 & $17.3^{* *}$ & & 10 & 09.0 & \\
\hline Unwanted Sexual Contact & & & & & & 0.263 & & & \\
\hline No & 232 & 93.5 & & 126 & 91.3 & & 106 & 96.4 & \\
\hline Yes & 16 & 6.5 & & 12 & 8.7 & & 4 & 3.6 & \\
\hline
\end{tabular}

${ }^{*} p \leq 0.001,{ }^{* *} p \leq 0.05$.

report being psychologically abused as adults if they had been physically abused as adults. Additionally, respondents were over six and half times as likely to report psychological abuse if they had been a child witness of intra-parental abuse (Exp. $\mathrm{B}=6.721, p \leq 0.000$ ). No statistically significant association was found with having been an adult victim of psychological abuse, or being a victim of attempted unwanted sexual contact or unwanted sexual contact. None of the child maltreatment variables-beat up, choked, sexual abuse, or threatened by a parent/caretaker achieved statistical significance. There was no statistically significant association with gender-men as well as women were more likely to experience psychological abuse if they had suffered physical abuse or if they had witnessed intra-parental abuse as a child (Table 5).

\section{Physical Abuse}

Three variables achieved a statistically significant association with being an adult victim of physical abuse. Respondents who had been beaten up by a parent/caregiver as a child were nearly two and a half times as likely to report physical abuse as an adult (Exp (B) 2.475). Respondents who reported suffering 
Table 5. Binomial regression of adult psychological abuse, by physical, and sexual abuse by child maltreatment, gender, and witnessing Intra-parental/caregiver abuse as a child $\mathrm{N}$ $=260$.

\begin{tabular}{|c|c|c|c|c|c|c|c|}
\hline & \multirow{2}{*}{ B } & \multirow{2}{*}{ S.E. } & \multirow{2}{*}{ Wald } & \multirow{2}{*}{$p$} & \multicolumn{3}{|c|}{ 95\% CI $\operatorname{Exp}(\mathrm{B})$} \\
\hline & & & & & Exp. (B) & Lower & Upper \\
\hline \multicolumn{8}{|l|}{ Before the age of $18 \mathrm{yrs:}$} \\
\hline Parent/caregiver choked you & 0.332 & 0.543 & 0.373 & 0.541 & 1.394 & 0.481 & 4.042 \\
\hline Parent/caregiver beat you up & -0.090 & 0.493 & 0.033 & 0.856 & 0.914 & 0.348 & 2.403 \\
\hline $\begin{array}{c}\text { Parent/caregivers } \\
\text { sexually abused you }\end{array}$ & 0.334 & 0.526 & 0.403 & 0.525 & 1.397 & 0.498 & 3.916 \\
\hline $\begin{array}{l}\text { Parent/caregivers } \\
\text { threatened you with a weapon }\end{array}$ & -0.554 & 0.553 & 1.005 & 0.316 & 0.574 & 0.194 & 1.698 \\
\hline $\begin{array}{c}\text { Witnessed } \\
\text { Intra-parental/caregiver Abuse }\end{array}$ & 1.905 & 0.341 & 31.188 & $0.000^{*}$ & 6.721 & 3.444 & 13.118 \\
\hline \multicolumn{8}{|l|}{ As an adult: } \\
\hline $\begin{array}{l}\text { Attempted unwanted } \\
\text { sexual contact }\end{array}$ & -0.198 & 0.538 & 0.135 & 0.713 & 0.821 & 0.286 & 2.357 \\
\hline Unwanted sexual contact & 0.968 & 0.599 & 2.617 & 0.106 & 2.633 & 0.815 & 8.511 \\
\hline Gender & 0.098 & 0.343 & 0.081 & 0.776 & 1.103 & 0.563 & 2.162 \\
\hline Adult Physical Abuse & 2.216 & 0.357 & 38.595 & $0.000^{*}$ & 9.172 & 4.558 & 18.454 \\
\hline Constant & -1.825 & 0.292 & 39.144 & 0.000 & 0.161 & & \\
\hline
\end{tabular}

${ }^{*} p \leq 0.000$.

psychological abuse as an adult were over eleven times as likely to be physically abused (Exp (B) 11.104). Women respondents were over twice as likely to report physical abuse as an adult (Exp (B) 2.286) (Table 6).

\section{Attempted Unwanted Sexual Contact}

A binomial regression analysis of an adult having experienced an attempted unwanted sexual contact by a partner found that having been sexually abused as a child by a parent/caregiver was statistically significantly associated with being an adult victim of an attempted unwanted sexual contact (Exp. (B) 3.563). Respondents who had been a victim of an unwanted sexual contact were nearly 11 times more at risk for being the victim of an attempted unwanted sexual contact (Exp. (B) 10.775). None of the other variables achieved a statistically significant association (Table 7).

Gender did not achieve statistical significance in this analysis. Recall that the cross tabulation showed that 14 men reported attempted or completed unwanted sexual contact while 36 women reported such events (Table 4).

\section{Unwanted Sexual Contact}

A binomial regression analysis of an adult having experienced an unwanted sexual contact found that having been sexually abused as a child by a parent/caregiver was statistically significantly associated with being an adult victim of an attempted unwanted sexual contact (Exp. (B) 10.775). None of the other variables achieved a statistically significant association (Table 8). 
Table 6. Binomial regression adult physical abuse by child maltreatment, witnessing intra-parental abuse as a child, gender, adult psychological abuse, and attempted unwanted sexual contact and unwanted sexual contact. $\mathrm{N}=260$.

\begin{tabular}{|c|c|c|c|c|c|c|c|}
\hline & \multirow{2}{*}{ B } & \multirow{2}{*}{ S.E. } & \multirow{2}{*}{ Wald } & \multirow{2}{*}{ Sig } & \multicolumn{3}{|c|}{ 95\% CI Exp (B) } \\
\hline & & & & & Exp. (B) & Lower & Upper \\
\hline \multicolumn{8}{|l|}{ Before the age of $18 \mathrm{yrs:}$} \\
\hline Parent/caregiver choked you & -0.293 & 0.533 & 0.303 & 0.582 & 0.746 & 0.262 & 2.121 \\
\hline Parent/caregiver beat you up & 0.906 & 0.417 & 4.729 & $0.030^{* *}$ & 2.475 & 1.094 & 5.600 \\
\hline $\begin{array}{l}\text { Parent/caregivers } \\
\text { threatened you with a weapon }\end{array}$ & 1.654 & 1.192 & 1.928 & 0.165 & 5.230 & 0.506 & 54.042 \\
\hline $\begin{array}{l}\text { Parent/caregivers } \\
\text { sexually abused you }\end{array}$ & 0.478 & 0.500 & 0.915 & 0.339 & 1.613 & 0.606 & 4.295 \\
\hline Witnessed Intra-parental abuse & 0.383 & 0.377 & 1.029 & 0.310 & 1.466 & 0.700 & 3.071 \\
\hline \multicolumn{8}{|l|}{ As an Adult: } \\
\hline $\begin{array}{l}\text { Attempted unwanted } \\
\text { sexual contact }\end{array}$ & 0.210 & 0.506 & 0.173 & 0.677 & 1.234 & 0.458 & 3.325 \\
\hline Unwanted sexual contact & 0.924 & 0.743 & 1.548 & 0.213 & 2.519 & 0.588 & 10.801 \\
\hline Gender & 0.827 & 0.327 & 6.378 & $0.012^{* *}$ & 2.286 & 1.203 & 4.344 \\
\hline Psychological abuse & 2.407 & 0.340 & 50.053 & $0.000^{*}$ & 11.104 & 5.700 & 21.632 \\
\hline Constant & -3.215 & 0.603 & 28.404 & 0.000 & 0.040 & & \\
\hline
\end{tabular}

${ }^{*} p \leq 0.000,{ }^{* *} p \leq 0.05$.

Table 7. Binomial regression adult attempted unwanted sexual contact by gender, adult physical psychological abuse, adult unwanted sexual contact, child maltreatment, and witnessing intra-parental abuse as a child $\mathrm{N}=260$.

\begin{tabular}{|c|c|c|c|c|c|c|c|}
\hline & \multirow{2}{*}{ B } & \multirow{2}{*}{ S.E. } & \multirow{2}{*}{ Wald } & \multirow{2}{*}{ Sig } & \multicolumn{3}{|c|}{ 95\% CI $\operatorname{Exp}(B)$} \\
\hline & & & & & Exp. (B) & Lower & Upper \\
\hline \multicolumn{8}{|l|}{ Before the age of $18 \mathrm{yrs:}$} \\
\hline Parent/caregiver choked you & 0.085 & 0.689 & 0.015 & 0.902 & 1.089 & 0.282 & 4.203 \\
\hline Parent/caregiver beat you up & -0.599 & 0.585 & 1.049 & 0.306 & 0.549 & 0.174 & 1.729 \\
\hline $\begin{array}{c}\text { Parent/caregivers sexually } \\
\text { abused you }\end{array}$ & 1.271 & 0.456 & 7.765 & $0.005^{* *}$ & 3.563 & 1.458 & 8.709 \\
\hline $\begin{array}{c}\text { Parent/caregivers threatened } \\
\text { you with a weapon }\end{array}$ & 0.226 & 0.712 & 0.101 & 0.751 & 1.254 & 0.310 & 5.062 \\
\hline $\begin{array}{c}\text { Witnessed } \\
\text { Intra-parental/caregiver abuse }\end{array}$ & 0.523 & 0.461 & 1.285 & 0.257 & 1.687 & 0.683 & 4.165 \\
\hline \multicolumn{8}{|l|}{ As an adult: } \\
\hline Gender & 0.491 & 0.448 & 1.203 & 0.273 & 1.634 & 0.680 & 3.930 \\
\hline Unwanted sexual contact & 2.377 & 0.626 & 14.412 & $0.000^{*}$ & 10.775 & 3.158 & 36.762 \\
\hline Physical abuse & 0.145 & 0.542 & 0.072 & 0.789 & 1.156 & 0.400 & 3.346 \\
\hline Psychological abuse & -0.111 & 0.499 & 0.050 & 0.824 & 0.895 & 0.337 & 2.378 \\
\hline Constant & -2.366 & 0.252 & 87.829 & -2.366 & 0.000 & & \\
\hline
\end{tabular}

${ }^{*} p \leq 0.000,{ }^{* *} p \leq 0.01$. 
Table 8. Binomial regression adult unwanted sexual contact by gender, adult physical and psychological abuse, adult attempted unwanted sexual contact, child maltreatment, and witnessing intra-parental abuse as a child $\mathrm{N}=260$.

\begin{tabular}{|c|c|c|c|c|c|c|c|}
\hline & \multirow{2}{*}{ B } & \multirow{2}{*}{ S.E. } & \multirow{2}{*}{ Wald } & \multirow{2}{*}{ Sig } & \multicolumn{3}{|c|}{ 95\% CI $\operatorname{Exp}(B)$} \\
\hline & & & & & Exp. (B) & Lower & Upper \\
\hline \multicolumn{8}{|l|}{ Before the age of $18 \mathrm{yrs}$ : } \\
\hline Parent/caregiver choked you & -18.377 & 7628.661 & 0.000 & 0.998 & 0.000 & 0.000 & \\
\hline Parent/caregiver beat you up & 0.059 & 0.755 & 0.006 & 0.938 & 1.061 & 0.241 & 4.661 \\
\hline $\begin{array}{c}\text { Parent/caregivers sexually } \\
\text { abused you }\end{array}$ & 1.167 & 0.647 & 3.247 & 0.072 & 3.211 & 0.903 & 11.424 \\
\hline $\begin{array}{c}\text { Parent/caregivers threatened } \\
\text { you with a weapon }\end{array}$ & 1.241 & 0.855 & 2.109 & 0.146 & 3.461 & 0.648 & 18.486 \\
\hline $\begin{array}{c}\text { Witnessed } \\
\text { Intra-parental/caregiver abuse }\end{array}$ & 0.343 & 0.780 & 0.194 & 0.660 & 1.409 & 0.306 & 6.496 \\
\hline \multicolumn{8}{|l|}{ As an adult: } \\
\hline Gender & -0.120 & 0.632 & 0.036 & 0.850 & 0.887 & 0.257 & 3.063 \\
\hline Attempted unwanted sexual contact & 2.377 & 0.626 & 14.412 & $0.000^{*}$ & 10.775 & 3.158 & 36.762 \\
\hline Physical abuse & -0.270 & 0.991 & 0.074 & 0.785 & 0.764 & 0.110 & 5.321 \\
\hline Psychological abuse & 0.739 & 0.674 & 1.201 & 0.273 & 2.093 & 0.559 & 7.846 \\
\hline Constant & -3.921 & 0.491 & 63.736 & 0.000 & 0.020 & & \\
\hline
\end{tabular}

${ }^{*} p \leq 0.000,{ }^{* *} p \leq 0.01$.

\section{Discussion}

The first aim of this study was to examine the extent of partner victimization and child maltreatment among a sample of college students and whether these experiences varied by gender. It is important to explore whether men experience partner victimization and child maltreatment. Despite the findings that women were statistically significantly more likely to experience certain types of victimization-this should not discount men's experiences. Consistent with previous research (Gover et al., 2008, Orcutt et al., 2005, Sabina \& Straus, 2008), college students in the current sample reported experiencing high rates of intimate violence at the hands of a partner. In contrast to most previous research, this study examined psychological and sexual victimization in addition to physical victimization and found that when all three are considered, more than half the sample reported at least one of the three types of abuse within the previous year. Findings revealed that women were more likely than men to report experiencing higher rates of psychological, physical and sexual abuse in their relationships, which is consistent with previous research (Gover et al., 2008; Harned, 2002). While over half of women respondents reported psychological abuse, nearly a third of the men surveyed experienced psychological abuse.

Similarly, a large number of respondents reported experiencing maltreatment as a child, which included physical abuse, sexual abuse, or witnessing abuse by a 
parent/caregiver. Psychological abuse experienced as a child (i.e., witnessing abuse) was the most prevalent form reported with more than one-third of the sample (37.1\%) experiencing such abuse. Consistent with previous research (Smith-Marek et al., 2015), findings in the current study revealed no significant differences between men and women experiences with childhood maltreatment, with the exception of sexual abuse by a parent/caregiver whereby women reported higher rates than men. Twenty-nine (20\%) women and nine (8\%) men reported sexual abuse as a child by a parent or caregiver. While more women were victimized, again, this should not negate men's victimization.

Consistent with previous research (Finkelhor et al., 2007a; Ford et al., 2013), results also showed that intimate partner violence rarely occurs in isolation. In the current, study those who reported psychological abuse in their adult relationships were over nine times more likely to report experiencing physical abuse by their partner. Additionally, respondents who reported physical abuse at the hands of their partner were over twice as likely to report sexual abuse as an adult. There were no statistically significant gender differences.

The second aim of the study was to explore whether polyvictimization increased the risk of adult intimate partner victimization. Despite growing interest concerning the correlates of childhood maltreatment and subsequent partner violence, few studies have examined polyvictimization using more than one category of child maltreatment or other forms of adult victimization experiences. Consistent with previous studies (Bensley, Van Eenwyk, \& Wynkoop Simmons, 2003; Gover et al., 2008; Langhinrichsen-Rohling, Hankla, \& Stormberg, 2004; Widom, Czaja, \& Dutton, 2014), our results found some support for the effects of polyvictimization and adult intimate partner victimization. Those respondents who reported as a child witnessing intra-parental/caregiver abuse and having been physically abused as an adult were more likely to report psychological abuse as an adult. Those respondents who had as a child been beat up by a parent/caregiver and suffered psychological abuse as an adult, and were women, were more likely to report physical abuse as an adult. While men were also victims of having been beat up as a child by a caregiver and suffered psychological abuse as a child, men were not statistically significant as likely to experience physical abuse as an adult.

In addition, respondents who had been victims of an attempted unwanted sexual contact by a partner also reported sexual abuse as a child by a parent/caregiver and also having been an adult victim of unwanted sexual contact. The only variable statistically significantly associated with unwanted sexual contact was having been a victim of attempted unwanted sexual contact. Gender was not statistically significantly associated with reporting unwanted sexual contact and experiencing attempted unwanted sexual contact. This was the only dependent variable that did not exhibit polyvictimization effects.

Although the current study extends our understanding of the correlates of partner victimization and child maltreatment, findings should be viewed with caution in light of several limitations. First, data were obtained by self-report. 
Thus, the possibility of deliberate response distortion must be considered. Second, present findings may not generalize beyond the particular sample. We note our sample consisted of a small number of college men and women from one institution who may differ from other groups in their experiences of abuse as adults and during childhood. The study does, however, provide evidence for future comparisons. Third, the cross-sectional design of this investigation does not allow causal inferences to be made regarding the temporal order of variables. Finally, the child maltreatment variables included in the current study were limited to certain experiences that occur in the home and do not represent a comprehensive list of early adversities that a child may experience. Finkelhor and colleagues (2015), for example, have suggested that associations between adverse childhood experiences and outcomes could be strengthened by including peer victimization, community violence, and socioeconomic status, to name a few. Future research should extend this study through the investigation of such variables.

The results of this study illustrate the importance of college health professionals to develop broader approaches when addressing intimate partner violence that includes outreach to both men and women. Consistent with previous studies (Clark et al., 1994; Harned, 2002; Finkelhor et al., 2007a, 2017b), this study indicates that men, as well as women, are victims of intimate partner violence and child maltreatment. Moreover, although not all children who witness violence or experience violence in the family of origin become victims of partner violence, the heightened risk of partner victimization makes it vital to consider the implications of child maltreatment for subsequent intimate relationships. Accordingly, when developing programs and services, college health professionals must strive for inclusivity, as well as develop targeted approaches for outreach to populations on their campuses that may be at greater risk.

The findings suggest that intimate partner violence among college students is a common occurrence. This fact should trigger institutions to develop more comprehensive means to both educate and address these concerns and should include all genders in their efforts. While results of the current study are retrospective, they still offer insight into the risk factors that are associated with intimate partner violence in college relationships. Accordingly, it is important that parents/caregivers and health professionals are aware of that violence witnessed as a child and abuse experienced as a child may have long term associations with adult victimization experiences.

\section{Conflicts of Interest}

The authors declare no conflicts of interest regarding the publication of this paper.

\section{References}

Aakvaag, H. F., Thoresen, S., Wentzel-Larsen, T., \& Dyb, G. (2017). Adult Victimization in Female Survivors of Childhood Violence and Abuse: The Contribution of Multiple 
Types of Violence. Violence against Women, 23, 1601-1619. https://doi.org/10.1177/1077801216664427

Akers, R. L. (1998). Social Learning and Social Structure: A General Theory of Crime and Deviance. Boston, MA: Northeastern University Press.

Bandura, A. (1973). Aggression: A Social Learning Analysis. Englewood Cliffs, NJ: Prentice-Hall. https://doi.org/10.2307/1227918

Bandura, A. (1977). Social Learning Theory. New York: General Learning Press.

Becker, K. D., Stuewing, J., \& McCloskey, L. A. (2010). Traumatic Stress Symptoms of Women Exposed to Different Forms of Childhood Victimization and Intimate Partner Violence. Journal of Interpersonal Violence, 25, 1699-1715. https://doi.org/10.1177/0886260509354578

Bensley, L., Van Eenwyk, J., \& Wynkoop Simmons, K. (2003). Childhood Family Violence History and Women's Risk for Intimate Partner Violence and Poor Health. American Journal of Preventive Medicine, 25, 38-44. https://doi.org/10.1016/S0749-3797(03)00094-1

Bookwala, J., Frieze, I. H., Smith, C., \& Ryan, K. (1992). Predictors of Dating Violence: A Multivariate Analysis. Violence and Victims, 7, 297-311. https://doi.org/10.1891/0886-6708.7.4.297

Brownridge, D. A. (2006). Partner Violence against Women with Disabilities. Violence against Women, 12, 805-822. https://doi.org/10.1177/1077801206292681

Clark, M., Beckett, J., Wells, M., \& Dungee-Anderson, E. (1994). Courtship Violence among African Americans. Journal of Black Psychology, 20, 264-281. https://doi.org/10.1177/00957984940203002

Colman, R. A., \& Widom, C. S. (2004). Childhood Abuse and Neglect and Adult Intimate Relationships: A Prospective Study. Child Abuse \& Neglect, 28, 1133-1151. https://doi.org/10.1016/j.chiabu.2004.02.005

Daigneault, I., Hebert, M., \& McDuff, P. (2009). Men's and Women's Childhood Sexual Abuse and Victimization in Adult Partner Relationships: A Study of Risk Factors. Child Abuse \& Neglect, 33, 638-647. https://doi.org/10.1016/j.chiabu.2009.04.003

DeKeseredy, W. S., Schwartz, M. D., Nolan, J., Mastron, N., \& Hall-Sanchez, A. (2019). Polyvictimization and the Continuum of Sexual Abuse at a College Campus: Does Negative Peer Support Increase the Likelihood of Multiple Victimizations? The British Journal of Criminology, 59, 276-295. https://doi.org/10.1093/bjc/azy036

Desai, S., Arias, I., \& Thompson, M. P. (2002). Childhood Victimization and Subsequent Adult Revictimization Assessed in a Nationally Representative Sample of Women and Men. Violence \& Victims, 17, 639-653. https://doi.org/10.1891/vivi.17.6.639.33725

Finkelhor, D., Ormrod, R. K., \& Turner, H. A. (2007a). Poly-Victimization: A Neglected Component in Child Victimization. Child Abuse \& Neglect, 31, 7-26. https://doi.org/10.1016/j.chiabu.2006.06.008

Finkelhor, D., Ormrod, R. K., \& Turner, H. A. (2007b). Re-Victimization Patterns in a National Longitudinal Sample of Children and Youth. Child Abuse \& Neglect, 31, 479-502. https://doi.org/10.1016/j.chiabu.2006.03.012

Finkelhor, D., Turner, H. A., Shattuck, A., \& Hamby, S. L. (2015). Prevalence of Childhood Exposure to Violence, Crime, and Abuse: Results from the National Survey of Children's Exposure to Violence. JAMA Pediatrics, 169, 746-754. https://doi.org/10.1001/jamapediatrics.2015.0676

Ford, J. D., Elhai, J. D., Connor, D. F., \& Frueh, B. C. (2010). Poly-Victimization and Risk of Posttraumatic, Depressive, and Substance Use Disorders and Involvement in Delin- 
quency in a National Sample of Adolescents. Journal of Adolescent Health, 46, 545-552. https://doi.org/10.1016/j.jadohealth.2009.11.212

Ford, J. D., Grasso, D. J., Hawke, J., \& Chapman, J. F. (2013). Poly-Victimization among Juvenile Justice-Involved Youths. Child Abuse and Neglect, 37, 788-800.

https://doi.org/10.1016/j.chiabu.2013.01.005

Foshee, V. A., Ennett, S. T., Bauman, K. E., Benefield, T., \& Suchindra, C. (2005). The Association between Family Violence and Adolescent Dating Violence Onset: Does It Vary by Race, Socioeconomic Status, and Family Structure? Journal of Early Adoles cence, 25, 317-344. https://doi.org/10.1177/0272431605277307

Gomez, A. M. (2011). Testing the Cycle of Violence Hypothesis: Child abuse and Adolescent Dating Violence as Predictors of Intimate Partner Violence in Adulthood. Youth \& Society, 43, 171-192. https://doi.org/10.1177/0044118X09358313

Gover, A., Kaukinen, C., \& Fox, K. A. (2008). The Relationship between Violence in the Family of Origin and Dating Violence among College Students. Journal of Interpersonal Violence, 23, 1667-1693. https://doi.org/10.1177/0886260508314330

Harned, M. S. (2002). A Multivariate Analysis of Risk Markers for Dating Violence Victimization. Journal of Interpersonal Violence, 17, 1179-1197.

https://doi.org/10.1177/088626002237401

Herrenkohl, T. I., Higgins, D. J., Merrick, M. T., \& Leeb, R. T. (2015). Positioning a Public Health Framework at the Intersection of Child Maltreatment and Intimate Partner Violence: Primary Prevention Requires Working outside Existing Systems. Child Abuse \& Neglect, 48, 22-28. https://doi.org/10.1016/j.chiabu.2015.04.013

Hines, D. A. (2007). Predictors of Sexual Coercion against Women and Men: A Multilevel, Multinational Study of University Students. Archives of Sexual Behavior, 36, 403-422. https://doi.org/10.1007/s10508-006-9141-4

Jennings, W. G., Park, M., Tomisich, E. A., Gover A. R., \& Akers R. L. (2011). Assessing the Overlap in Dating Violence Perpetration and Victimization among South Korean College Students: The Influence of Social Learning and Self-control. American Journal of Criminal Justice, 36, 188-206. https://doi.org/10.1007/s12103-011-9110-x

Kaukinen, C., Buchanan, L., \& Gover, A. R. (2015). Child Abuse and the Experience of Violence in College Dating Relationships: Examining the Moderating Effect Offender and Race. Journal of Family Violence, 30, 1079-1092.

https://doi.org/10.1007/s10896-015-9731-9

Langhinrichsen-Rohling, J., Hankla, M., \& Stromberg, C. D. (2004). The Relationship Behavior Networks of Young Adults: A Test of the Intergenerational Transmission of Violence Hypothesis. Journal of Family Violence, 19, 139-151. https://doi.org/10.1023/B:JOFV.0000028074.35688.4f

Marshall, L. L., \& Rose, P. (1988). Family of Origin Violence and Courtship Abuse. Journal of Counseling and Development, 66, 414-418.

https://doi.org/10.1002/j.1556-6676.1988.tb00902.x

Millett, L. S., Kohl, P.L., Jonson-Reid, M., Drake, B., \& Petra, M. (2013). Child Maltreatment Victimization and Subsequent Perpetration of Young Adult Intimate Partner Violence: An Exploration of Mediating Factors. Child Maltreatment, 18, 71-84. https://doi.org/10.1177/1077559513484821

Orcutt, H. K., Marilyn, G., \& Pickett, S. M. (2005). Female-Perpetrated Intimate Partner Violence and Romantic Attachment Style in a College Student Sample. Violence and Victims, 20, 287-302. https://doi.org/10.1891/vivi.20.3.287

Peterson, C., Florence, C., \& Klevens, J. (2018). The Economic Burden of Child Maltreatment in the United States, 2015. Child Abuse \& Neglect, 86, 178-183. 
https://doi.org/10.1016/j.chiabu.2018.09.018

Ports, K. A., Ford, D. C., \& Merrick, M. T. (2016). Adverse Childhood Experiences and Sexual Victimization in Adulthood. Child Abuse \& Neglect, 51, 313-322. https://doi.org/10.1016/j.chiabu.2015.08.017

Renner, L. M., \& Slack, K. S. (2006). Intimate Partner Violence and Child Maltreatment: Understanding Intra-and Intergenerational Connections. Child Abuse \& Neglect, 30, 599-617. https://doi.org/10.1016/j.chiabu.2005.12.005

Richards, T. N., Tillyer, M. S., \& Wright, E. M. (2017). Intimate Partner Violence and the Overlap of Perpetration and Victimization: Considering the Influence of Physical, Sexual, and Emotional abuse in Childhood. Child Abuse \& Neglect, 67, 240-248. https://doi.org/10.1016/j.chiabu.2017.02.037

Richmond, J. M., Elliott, A. E., Pierce, T. W., Aspelmeier, J. E., \& Alexander, A. A. (2009). Polyvictimization, Childhood Victimization, and Psychological Distress in College Women. Child Maltreatment, 14, 127-147. https://doi.org/10.1177/1077559508326357

Riggs, D. S., Caulfield, M. B., \& Fair, K. (2009). Risk of Intimate Partner Violence: Factors Associated with Perpetration and Victimization. In P. M. Kleespies (Ed.), Behavioral Emergencies: An Evidence-Based Resource for Evaluating and Managing Risk of Suicide, Violence, and Victimization (pp. 189-208). Washington DC: American Psychological Association. https://doi.org/10.1037/11865-009

Sabina, C., \& Straus, M. A. (2008). Polyvictimization by Dating Partners and Mental Health among U.S. College Students. Violence and Victims, 23, 667-682.

https://doi.org/10.1891/0886-6708.23.6.667

Smith-Marek, E. N., Cafferky, B., Dharnidharka, P., Mallory, A. B., Domínguez, M., High, J., Stith, S., \& Méndez, M. (2015). Effects of Childhood Experiences of Family Violence on Adult Partner Violence: A Meta-Analytic Review. Journal of Family Theory and Review, 7, 498-519. https://doi.org/10.1111/jftr.12113

Stets, J. E., \& Henderson, D. (1991). Contextual Factors Surrounding Conflict Resolution While Dating: Results from a National Study. Family Relations, 40, 29-36. https://doi.org/10.2307/585655

Stevens, A. L., Herrenkohl, T. I., Mason, W. A., Smith, G. L., Klevens, J., \& Merrick, M. T. (2018). Developmental Effects of Childhood Household Adversity, Transitions, and Relationship Quality on Adult Outcomes of Socioeconomic Status: Effects of Substantiated Child Maltreatment. Child Abuse \& Neglect, 79, 42-50. https://doi.org/10.1016/j.chiabu.2018.01.031

Stith, S. M., Rosen, K. H., Middleton, K. A., Busch, A. L., Lundeberg, K., \& Carlton, R. P. (2000). The Intergenerational Transmission of Spouse Abuse: A Meta-Analysis. Journal of Marriage and Family, 62, 640-654. https://doi.org/10.1111/j.1741-3737.2000.00640.x

Straus, M. A., Hamby, S. L., Boney-McCoy, S., \& Sugarman, D. B. (1996). The Revised Conflict Tactics Scale (CTS2): Development and Preliminary Psychometric Data. Journal of Family Issues, 17, 283-316. https://doi.org/10.1177/019251396017003001

U.S. Department of Health \& Human Services, Administration for Children and Families, Administration on Children, Youth and Families, Children's Bureau (2018). Child maltreatment, 2016. https://www.acf.hhs.gov/sites/default/files/cb/cm2016.pdf

Widom, C. S., Czaja, S. J., Bentley, T., \& Johnson, M. S. (2012). A Prospective Investigation of Physical Health Outcomes in Abused and Neglected Children: New Findings from a 30-Year Follow-Up. American Journal of Public Health, 102, 1135-1144. https://doi.org/10.2105/AJPH.2011.300636

Widom, S. J., Czaja, S., \& Dutton, M. A. (2014). Child Abuse and Neglect and Intimate Partner Violence Victimization and Perpetration: A Prospective Investigation. Child Abuse \& Neglect, 38, 650-663. https://doi.org/10.1016/j.chiabu.2013.11.004 